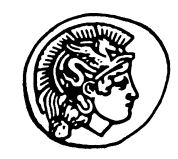

PERGAMON
Journal of Geodynamics 31 (2001) 459-479
JOURNAL OF

GEODYNAMICS

www.elsevier.nl/locate/jgeodyn

\title{
On the advection of sharp material interfaces in geodynamic problems: entrainment of the $\mathrm{D}^{\prime \prime}$ layer
}

\author{
Farhad Sobouti $^{\mathrm{a}, *}$, Abdolreza Ghods ${ }^{\mathrm{b}}$, Jafar Arkani-Hamed ${ }^{\mathrm{a}}$ \\ ${ }^{a}$ Earth and Planetary Sciences, McGill University, Montréal, QC H3A 2A7, Canada \\ ${ }^{\mathrm{b}}$ Department of Physics, Institute for Advanced Studies in Basic Sciences, Zanjan, Iran
}

Received 9 January 2001; received in revised form 6 April 2001; accepted 16 April 2001

\begin{abstract}
The $\mathrm{D}^{\prime \prime}$ layer is a dense and chemically distinct layer at the base of the convecting mantle. Numerical modeling of the entrainment of this layer by mantle convection requires the solution of the advective transport equation without introducing numerical diffusion across sharp material boundaries. We use our improved second moment numerical method to solve the equation. The method conserves the amount of material and the first and second moments of material distribution in each control volume. We first consider two examples of isothermal Rayleigh-Taylor instability to illustrate the performance of our method by comparing our results with those of a number of field, tracer and marker chain methods. We show that the performance of our method in minimizing the numerical diffusion is better than the field methods and comparable to the tracer and marker chain methods. We then study the instability of the dense $\mathrm{D}^{\prime \prime}$ layer and its interaction with the overlying mantle. A range of density contrast between the $\mathrm{D}^{\prime \prime}$ layer and the mantle, layer thickness, and the Rayleigh number, $R a$, is examined. We show that for higher values of these parameters, the amount of entrainment decreases and the layer remains stable over longer periods of time. For very thick $\mathrm{D}^{\prime \prime}$ layers and high $R a$ values, internal convection can take place within the layer. (C) 2001 Elsevier Science Ltd. All rights reserved.
\end{abstract}

\section{Introduction}

The heterogeneous nature of the Earth's chemical structure makes the study of compositionally heterogeneous flows of prime importance for the understanding of a wide range of geodynamic processes. Examples include, evolution of chemical plumes and entrainment of mantle material in them (Kellogg and King, 1997), salt diapirism (van Keken et al., 1993), mixing and distribution of

* Corresponding author. Fax: + 1-514-398-4680.

E-mail address: farhads@eps.mcgill.ca (F. Sobouti). 
geochemical heterogeneities in the mantle(Kellogg and Turcotte, 1990; Ferrachat and Ricard, 1998), segregation of lithospheric components in the convecting mantle (Christensen and Hofmann, 1994), effects of chemical buoyancy on the dynamics of subducting slabs in the transition zone (Christensen, 1997), entrainment of the $\mathrm{D}^{\prime \prime}$ layer by thermo-chemical convection (Hansen and Yuen, 1989; Montague and Kellogg, 2000), and the role of near-surface chemical boundary layer in mantle convection (Lenardic and Kaula, 1996).

The $\mathrm{D}^{\prime \prime}$ layer is a region of great seismic complexity (Lay et al., 1998), that points towards significant variations in its thickness and chemical composition. The $\mathrm{D}^{\prime \prime}$ layer is a dense compositional boundary layer embedded inside the thermal boundary layer at the core-mantle boundary (CMB). The entrainment of the layer can have important consequences for thermo-chemical instabilities near the base of the mantle. Numerical models that consider only a simple thermal boundary layer at the CMB fail to accurately explain the variations of the layer as inferred from seismic studies (Montague and Kellogg, 2000). Our objective is to determine whether the $\mathrm{D}^{\prime \prime}$ layer is a permanent feature at the $\mathrm{CMB}$, or it can become entrained by mantle convection. The entrainment is a long-term process that involves extensive mixing of chemically-heterogeneous materials in a convective system. The accumulated diffusion errors introduced by inadequate numerical schemes can ultimately render the results unreliable. Therefore, the accuracy of the numerical method is crucial for modeling of the evolution of the $\mathrm{D}^{\prime \prime}$ layer.

A major challenge in numerical modeling of heterogeneous flow problems is the accurate representation of material discontinuities. Solving the advection equation to trace sharp material interfaces often result in localized numerical diffusion and dispersion errors that slow the convergence rate of the solutions and may ultimately degrade the accuracy of the results (Lenardic and Kaula, 1993). In solid Earth geodynamics, most of the numerical techniques used in modeling chemical heterogeneities fall into two major categories; the field methods and the tracer particle methods. In the field methods the chemical compositions are represented by continuous functions and are advected through the flow field by solving the transport equation. An important factor in the accuracy of the field methods is the order of the numerical scheme used. Loworder schemes often suffer from numerical diffusion due to truncation errors, while high-order schemes may result in unphysical oscillations in the regions near material interfaces. Therefore, corrective measures are needed to suppress the oscillatory behavior of high-order schemes. These corrective techniques include the use of, for example, numerical filters (Lenardic and Kaula, 1993) and flux limiters (Sweby, 1984). In the tracer particle methods, the compositionally distinct domains are represented by a number of tracers, each having an independent concentration. The tracers are advected through the flow in a Lagrangian way and the compositional fields are calculated from the local tracer density (e.g. Christensen and Hofmann, 1994). In the marker chain method the tracers are used to define the sharp interface between different chemical domains (e.g. Woidt, 1978). The evolution of the interface is determined by displacing the markers along the instantaneous stream lines. The tracer methods have one major drawback, that is, spurious tracer accumulations often result in incorrect distribution of material in the flow. Therefore, care should be taken in choosing the number of tracers in order to avoid such inaccuracies. Also, the exponential growth of the marker chains in complex flow fields could result in termination of the numerical calculations (e.g. van Keken et al., 1997).

Egan and Mahoney (1972) introduced the second moment method to solve the incompressible advective-diffusive transport equation. The method advects the material discontinuities by conserving 
the mass and the first and second moments of material distribution inside each control volume. It is a conservative, explicit forward-in-time method, and can be regarded as quasi-Lagrangian in the sense that the material distribution in each control volume is advected along the streamlines over a time step. The advantage of this method is that numerical diffusion is minimized without producing numerical oscillations. For pure translational flows the method is shape preserving. However, its accuracy diminishes for compressional, rotational and shear deformation. In this paper we use our numerical method (Ghods et al., 2000) which is an improved version of the second moment method.

We first investigate two Rayleigh-Taylor (RT) instability problems using our method and two second-order-accurate field methods, one with a numerical filter (Lenardic and Kaula, 1993) and another with a flux limiter (Sweby, 1984). Of particular interest are the amount of numerical diffusion and the ability of the methods in estimating the global as well as detailed, local characteristics of the problems. We then study the dynamics of the $\mathrm{D}^{\prime \prime}$ layer through a suit of models that examine the effects of density contrast between the layer and the overlying mantle, the layer thickness, and the Rayleigh number on the entrainment of the layer by thermal convection.

\section{Governing equations and numerical methods}

We consider a Boussinesq fluid with an infinite Prandtl number containing two distinct chemical components. The non-dimensional governing equations in a two-dimensional rectangular coordinate system are:

conservation of mass

$$
\nabla \cdot v=0
$$

conservation of momentum

$$
\nabla \cdot \tau-\nabla P=\left(\begin{array}{lll}
R a & T-R b & C
\end{array}\right) \vec{z}
$$

conservation of energy

$$
\frac{\partial T}{\partial t}+v \cdot \nabla T=\nabla^{2} T
$$

and advection of chemical composition

$$
\frac{\partial C}{\partial t}+v \cdot \nabla C=0
$$

where $R a=\frac{\alpha \rho g \Delta T d^{3}}{\kappa \eta_{\mathrm{r}}}$ and $R b=\frac{\Delta \rho g d^{3}}{\kappa \eta_{\mathrm{r}}}$ are the thermal and compositional Rayleigh numbers, respectively. $v$ is the velocity vector, $\tau$ is the deviatoric stress tensor, $P$ is the dynamic pressure, $T$ is the temperature, $C$ is the composition function, $t$ is time, $\vec{z}$ is the unit vector in the direction of gravity, $\alpha$ is the thermal expansion coefficient, $g$ is the gravitational acceleration, $\rho$ is the density, 
$\Delta \rho$ is the density difference between the two components, $\Delta T$ is the temperature change from top to bottom of the model, $d$ is the depth, $\eta_{\mathrm{r}}$ is the reference viscosity and $\kappa$ is the thermal diffusivity. The composition function $C$ is initially a step function having values of zero and one, each corresponding to one of the components. The normalization parameters are chosen as follows: length, $d$; time, $d^{2} / \kappa$; temperature, $\Delta T$ and viscosity, $\eta_{\mathrm{r}}$. In Eq. (4) we have neglected chemical diffusion and considered the transport of compositional differences to be purely advective.

We used an iterative finite volume method to solve the equations of motion, conservation of mass and energy in primitive variable formulation. Second order central-difference scheme is used for the discretization of the velocity field. The temperature field is discretized by a hybrid method consisting of central-difference and weighted upwind schemes (Patankar, 1980). The method produces diffusion error which is small compared to thermal diffusion. The temperature field is integrated forward in time using fourth order Runge-Kutta method. To ensure reliability of our numerical model, we reproduced some of the benchmark solutions reported by Blankenbach et al. (1989).

Here we briefly describe our method of solving the advection equation (see Ghods et al., 2000 for details). A concentration field $C$ is represented by a number of discrete rectangular boxes, one box in each control volume (Fig. 1). Each rectangular distribution has a mean concentration $\left(C_{\mathrm{m}}\right)$, a center of concentration (with $x, y$ coordinates of $F_{x}$ and $F_{y}$ ) denoting the first moment of concentration distribution with respect to the center of the control volume, and dimensions $\left(R_{x}\right.$ and $R_{y}$ ) proportional to the square root of the second moment of concentration distribution with respect to the center of concentration distribution. In a control volume of dimensions $1 \times 1$ these quantities are calculated as:

$$
\begin{aligned}
& C_{\mathrm{m}}=\int_{-0.5}^{0.5} \int_{-0.5}^{0.5} C(x, y) \quad \mathrm{d} x \quad \mathrm{~d} y \\
& F_{x}=\frac{1}{C_{\mathrm{m}}} \int_{-0.5}^{0.5} \int_{-0.5}^{0.5} x \quad C(x, y) \quad \mathrm{d} x \quad \mathrm{~d} y \\
& F y=\frac{1}{C_{\mathrm{m}}} \int_{-0.5}^{0.5} \int_{-0.5}^{0.5} y \quad \mathrm{C}(\mathrm{x}, \mathrm{y}) \quad \mathrm{dx} \quad \mathrm{dy} \\
& R_{x}^{2}=\frac{1}{C_{\mathrm{m}}} \int_{-0.5}^{0.5} \int_{-0.5}^{0.5}\left(x-F_{x}\right)^{2} C(x, y) \quad \mathrm{d} x \quad \mathrm{~d} y \\
& R_{y}^{2}=\frac{1}{C_{\mathrm{m}}} \int_{-0.5}^{0.5} \int_{-0.5}^{0.5}(y-F y)^{2} C(x, y) \quad \mathrm{d} x \quad \mathrm{~d} y .
\end{aligned}
$$

During a time step, the boxes are moved as rigid bodies within and across the control volumes with the respective nodal velocities, and are partitioned appropriately between control volumes. At the end of the time step, the total mass and the first and second moments of all portions of the rectangular boxes in a given control volume are calculated and a single rectangular box is 


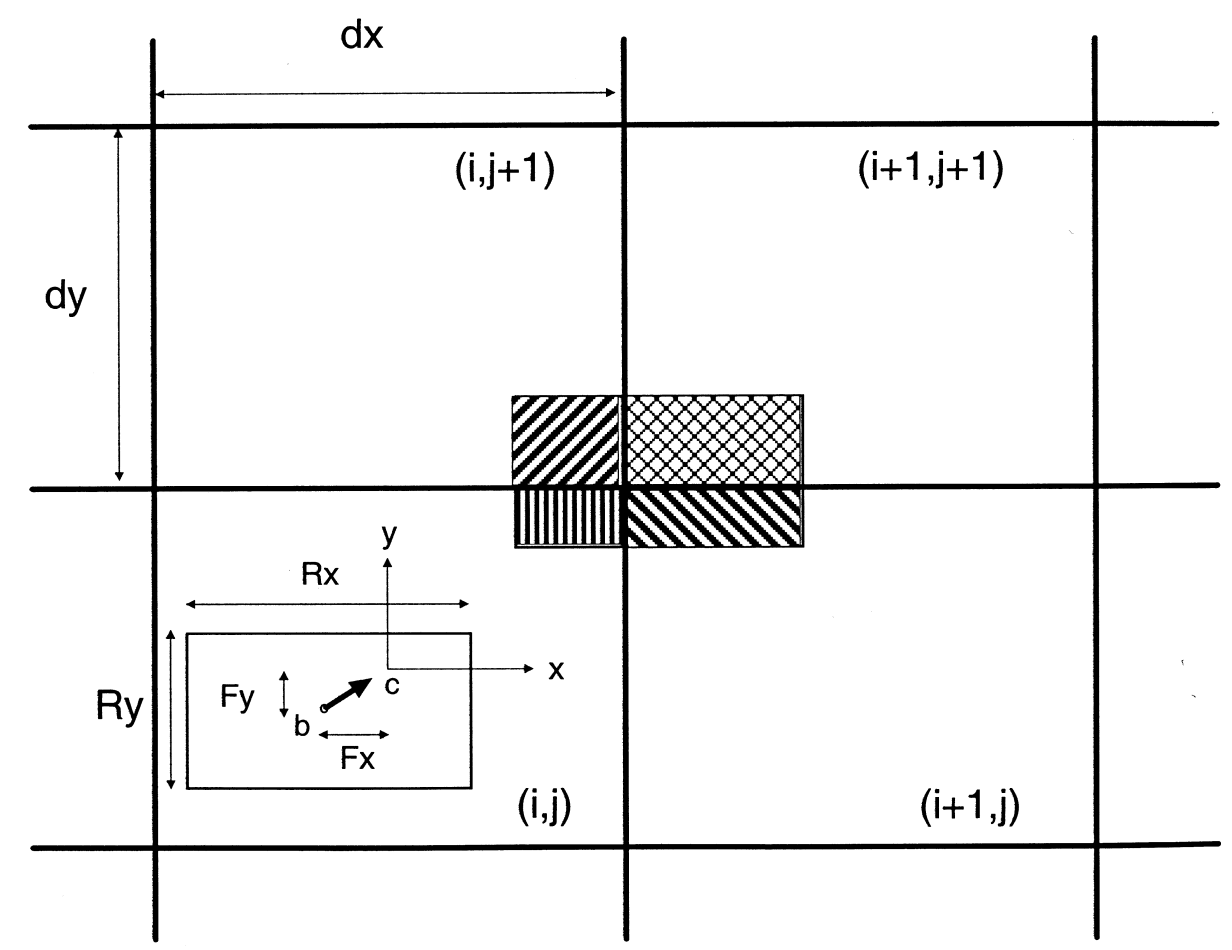

Fig. 1. Representation of the concentration field in control volume $(i, j)$ as a rectangular box (blank rectangle). The arrow denotes the velocity vector of the box. Points $b$ and $c$ denote the center of the box and the center of the control volume, respectively. The patterned box shows the partition of the advected box between the downstream control volumes.

constructed inside the control volume with the same amount of material and identical first and second moments. This adjustment of material distribution at the end of the time step serves to minimize numerical diffusion and essentially preserves the original shape of the concentration distribution.

In pure translational flows where fluid particles do not undergo any deformation, the assumption of rigid rectangular boxes is valid to a high degree of accuracy and keeps diffusion at a minimal level. However, in dilatational and shear deformation, the rigidity of the boxes does not hold and significant errors may occur in the calculation of the position and volume of the boxes, if Egan and Mahoney's method is used. Consequently, considerable diffusion error and unrealistic mass distributions such as overlapping of the rectangular boxes and accumulation of material at stagnation points can occur. Our method partially alleviates these shortcomings in two parts. In the first part, the assumption of the rigidity of the boxes is relaxed in order to accommodate the volumetric deformation in the flow field. In the second part, the dimensions of the rectangular boxes are adjusted to preserve their volume. The first improvement is accomplished by advecting each side of a given rectangular box with its particular velocity relative to the velocity of the center of the box. The sides of a box are advected parallel to the coordinate axes, and the effect of shape change due to shear deformation is not taken into account. Therefore, this correction accounts only for dilatational deformation and the boxes remain rectangular. The second 
improvement minimizes false diffusion associated with errors in the calculation of the total volume of rectangular boxes in a control volume. These errors arise when the distribution of the concentration in a given control volume assumes complex shapes, such as overlapping boxes.

Two other methods are used for comparison: the monotonic second order upwind (MSOU) method developed by Sweby (1984), and the second order upwind method combined with Lenardic and Kaula's (1993) global filter (FSOU). MSOU is a nearly second-order-accurate field method that uses quadratic upwind interpolation for representation of composition and a flux limiter to eliminate numerical oscillatory errors. Tamamidis and Assanis (1993) compared the performance of MSOU with other high-order-accuracy schemes and concluded that it is one of the most accurate field methods in problems involving advection of sharp discontinuities. The filter used in FSOU is global and corrects the nodal values of the concentration function irrespective of the neighboring grid points. An advantage of the filter is its ease of implementation.

\section{Isothermal RT instability of a thin dense layer}

There are many examples of geophysical and geochemical processes in the Earth's interior that give rise to a thin dense layer atop of a lighter region, leading to the Rayleigh-Taylor instability of the layer. For example, the chemical differentiation, crystallization and solidification of magma ocean in the early history of the Earth may have resulted in a late-stage ilmenite-rich cumulate layer with a high concentration of radioactive elements and iron at shallow depths. This dense layer is susceptible to gravitational instability and eventually sinks into the deeper interior. A similar process has recently been suggested by Hess and Parmentier (1995) to provide heat source for partial melting in the deeper parts of the Moon and generate the lunar high-Ti basalts.

Here we investigate the isothermal Rayleigh-Taylor instability of a thin dense fluid layer overlying a lighter fluid. The aim of our experiment is to study the diffusion error produced by the numerical methods at the material interface as the dense fluid penetrates down into the lighter one. Lenardic and Kaula (1993) studied this problem and we use their model specifications. A $52 \times 52$ element uniform grid is used with the top four element rows initially assigned to the dense layer. The initial distortion of the interface is produced by adding a $3 \times 3$ element region to the base of the dense layer at the left hand corner. Free-slip and impermeable boundary conditions are used on all boundaries. The compositional Rayleigh number is $R b=10^{6}$, and the viscosity of the two fluids is assumed to be equal.

When the interface between the two layers is distorted, small horizontal gradients of pressure are generated which induce motions in the two fluids that further deform the interface, and the thinner layer eventually evolves into a plume structure (Whitehead and Luther, 1975). Laboratory and numerical experiments on a plume moving through a layer of different density (Whitehead and Luther, 1975; Woidt, 1978) have shown that the plume is characterized by a spoutshape head with a vortex ring structure in the head of the spout that entrains the surrounding material, and the tail of the spout continuously feeds the head.

Fig. 2 shows the evolution of the compositional field of the dense layer calculated by FSOU, MSOU and our method (ISMM). In all three methods the plume develops a mushroom structure as it descends. FSOU (Figs. 2a and b and 3c) produces the greatest amount of diffusion, which results in a large plume head, whereas our method (Fig. $2 \mathrm{~g}, \mathrm{~h}$ and i) has the smallest plume head 
FSOU
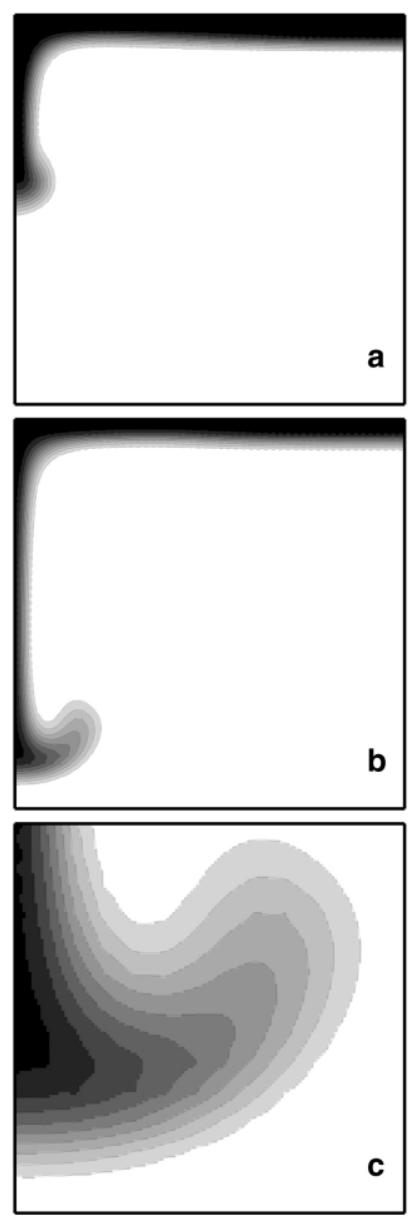

MSOU
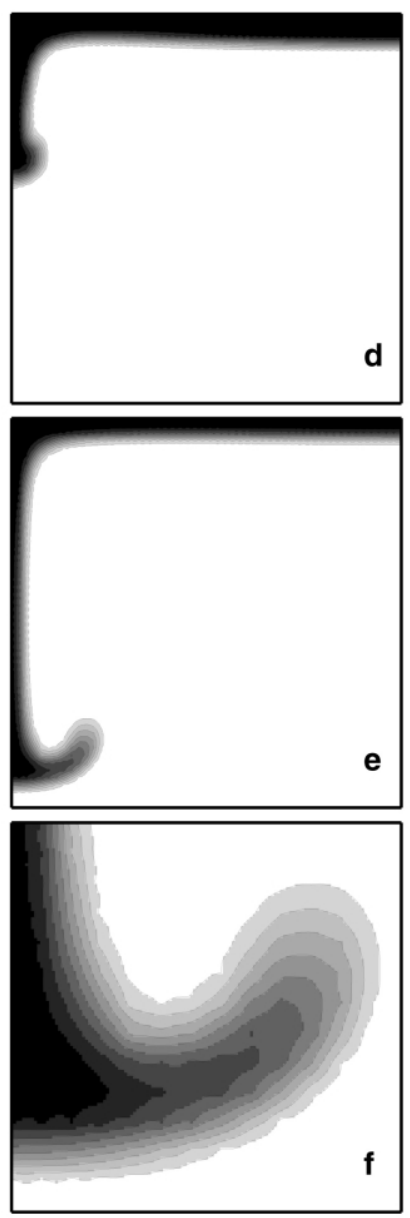

ISMM
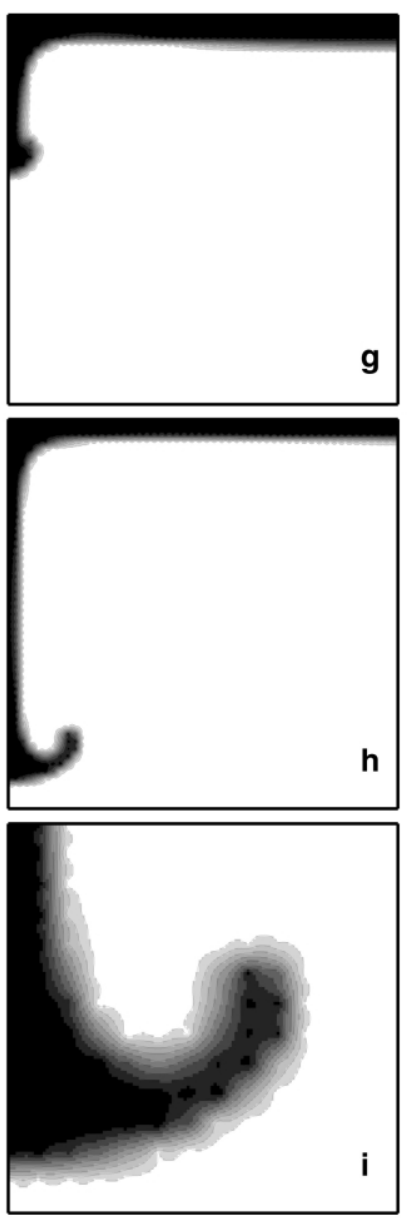

Fig. 2. The evolution of the compositional field for the RT instability of a dense layer. The plots of the third row are blow-ups of the plume head in the second row. a, b and c are for FSOU; $\mathrm{d}$, e and f are for MSOU; and g, h and i are for our method (ISMM).

comprised mostly of the undiffused dense fluid. Fig. 3 shows the profiles of composition for the three methods along the left boundary at equal time intervals as the plume descends. The FSOU and MSOU methods do not maintain the steep gradient of the material interface. In the case of FSOU the interface is resolved over about eight grid points, and for MSOU over six grid points. In contrast, our method (Fig. 3c) is very efficient in maintaining the sharpness of the interface, and diffusion spreads over only three grid points. Our method results in some enhancement of the compositional field in front of the plume, which can be substantially suppressed by reducing the time steps for only the advective transport equation. This increases the computation time, but since smaller time steps increase the convergence rate, the net effect of reduced time steps is not always prohibitive. The profile in Fig. $3 \mathrm{~d}$ shows the result when the time steps of the advection equation are reduced by two orders of magnitude. To illustrate the overall efficiency of the 

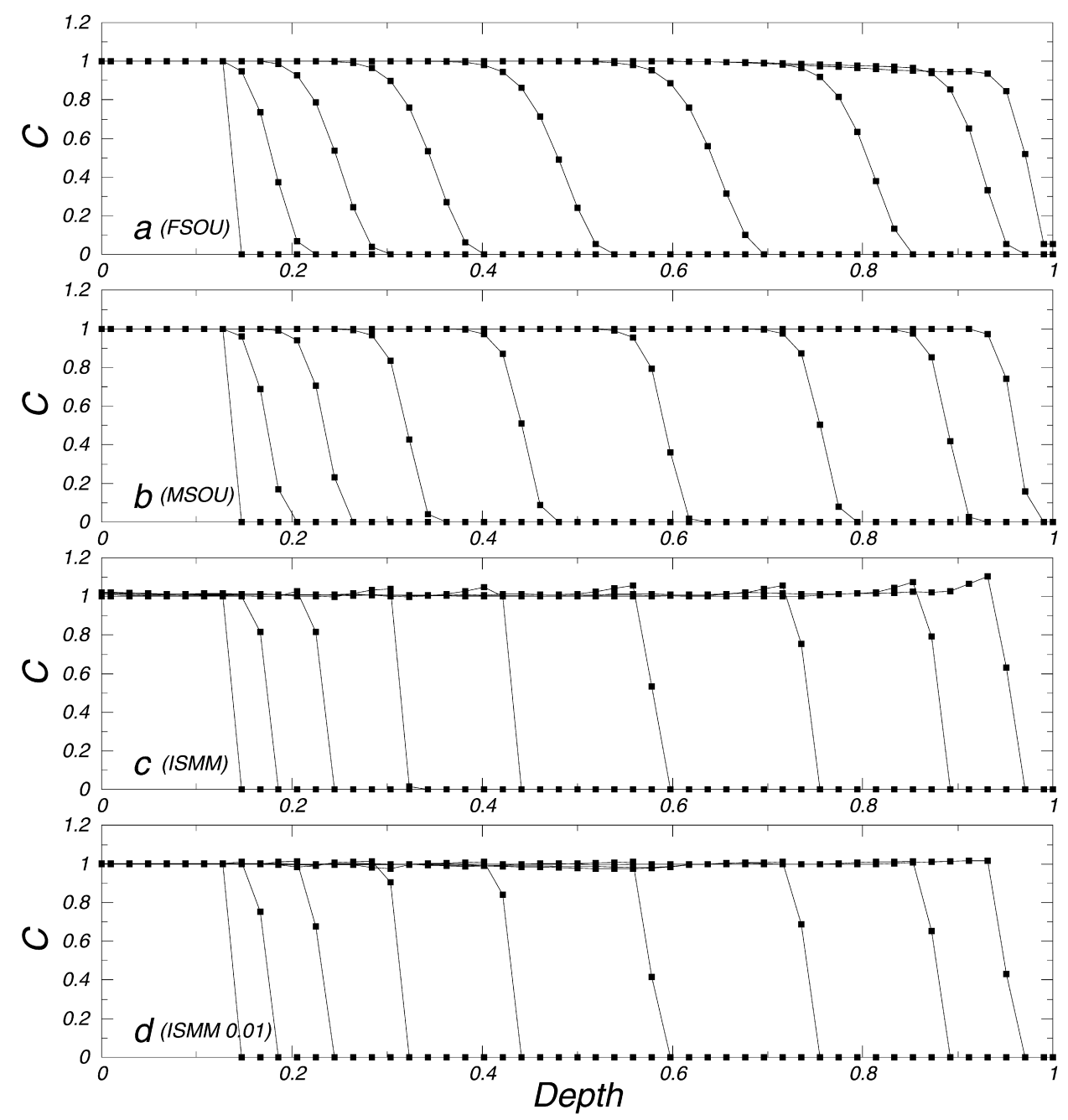

Fig. 3. Profiles of composition along the left boundary of the model with a dense unstable layer. In all models the total non-dimensional time is $t=4 \times 10^{-4}$ and profiles are shown at equal time intervals of $1 / 8$ of the total time. The first profile on the left in each figure corresponds to the initial $C$ field. The solid squares denote the positions of the grid points along the profiles. Fig. $d$ is obtained by our method with the time step for the advection equation reduced by a factor of 100 .

methods in minimizing the numerical diffusion we compare the average nodal resolving power of the three methods (Fig. 4). This parameter is defined as the total number of grid points with $C$ values between 0 and 1 divided by the number of grid columns (Lenardic and Kaula, 1993). The resolving power of FSOU and MSOU decreases as time proceeds, with MSOU having a slightly better result than FSOU. The resolving power of our method quickly reaches its minimum, and remains almost constant throughout the experiment and is always much stronger than FSOU and MSOU. These features suggest that the numerical diffusion of our method does not increase in time, whereas in the other two methods the boundaries of the plume are increasingly smeared out as the plume is advected. 


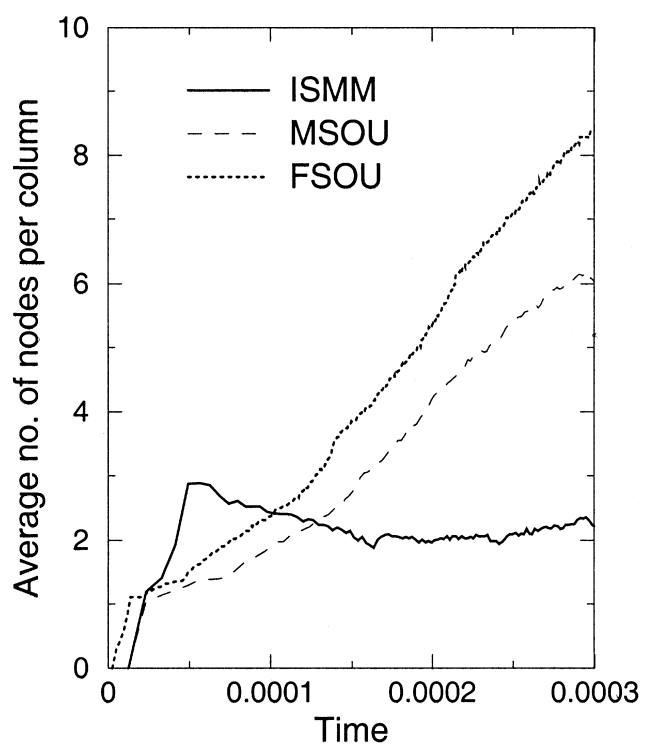

Fig. 4. Average nodal resolving power of the various methods for the RT instability of a dense layer.

\section{Isothermal RT instability of a thin light layer}

This kind of Rayleigh-Taylor instability also exists in the Earth. For example, the solidification of the inner core enhances the content of the light elements immediately above the inner coreouter core interface because they do not get incorporated in the solid inner core. Consequently, a low density thin layer is produced that underlies the denser outer core. The non-thermal convection resulting from the gravitational instability of this layer is shown to be the main source of the energy for maintaining the core dynamo (Braginski, 1963). This is a well recognized RayleighTaylor instability that occurs in the deeper interior of the Earth. Another example is the ascent of light salt domes through denser sedimentary layers.

van Keken et al. (1997) studied the Rayleigh-Taylor instability of a thin light layer. We adopt their model properties for easy comparison and assume a box of aspect ratio (width/depth) $\lambda=0.9142$ with a lighter layer of thickness $d_{\mathrm{b}}=0.2$ at the bottom. The compositional Rayleigh number is $R b=1$ and the amplitude of the initial perturbation of the interface is defined by $w=0.02 \cos (\pi x / \lambda)$. The top and bottom boundaries are rigid, and the side boundaries are free-slip and impermeable. We consider a case of isoviscous materials. van Keken et al. (1997) used four different methods to study this problem: a field method with second order upwind interpolation together with a numerical filter (their SK method), a marker chain method (PvK), and two tracer methods (HS and CND). We present the results of our computations and also compare them with those of van Keken et al. (1997) to demonstrate the ability of our method in determining the global parameters of the flow.

Fig. 5 shows the time evolution of the compositional field of the model using our method, as well as the results at $t=2000$ calculated by FSOU and MSOU. The development of the primary diapir (along the left boundary) and the secondary diapir (along the right boundary) is similar in all models, and they exhibit analogous features of the sinking dense-fluid plumes we discussed 

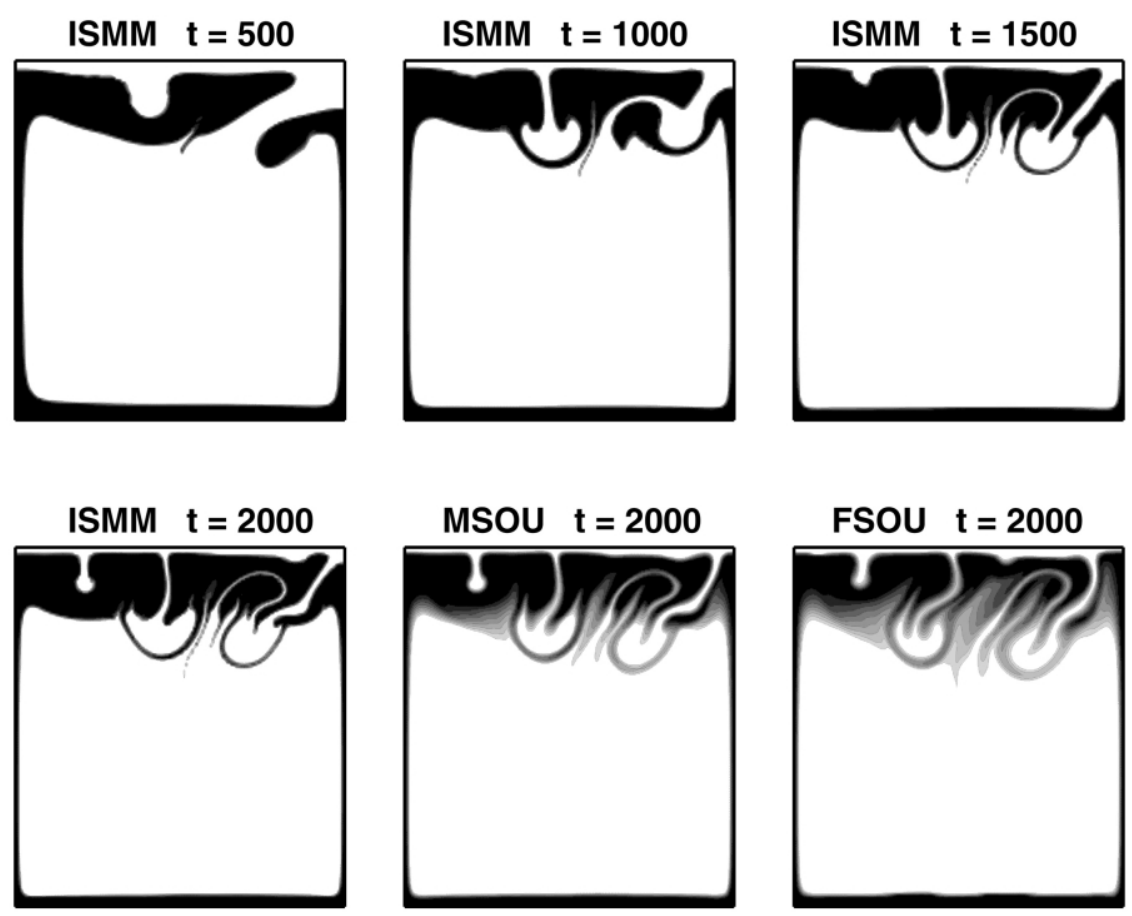

Fig. 5. Time evolution of the composition field for the isothermal RT instability of a light layer, obtained on a grid of $160 \times 160$ elements. Dark colors denote the lighter material. The plots show the composition field at time intervals of $t=500$ determined by our method, as well as that calculated by FSOU and MSOU at $t=2000$. Contour interval is 0.1 .

earlier. Therefore, we consider the later stages of the model. The presence of a thin dense layer at the top and a thin light layer at the bottom is due to the rigid velocity boundary conditions. The difference in the sharpness of the material interface is significant, which is also evident from the average resolving powers of Fig. 6. FSOU and MSOU have considerably diffused interfaces that further deteriorate with time. In our method, the interface in most places is resolved over three or four grid points, and the sharpness of the interface is kept at an almost constant level throughout the computation time. The resolving power of our method is greater than that of FSOU by more than a factor of two and almost twice as great as that of MSOU.

Fig. $7 \mathrm{a}$ and $\mathrm{b}$ shows the time variations of the root-mean-square velocity, $V_{\mathrm{rms}}$, and the entrainment of the lighter layer above the initial interface, $e$, defined as:

$$
e=\frac{1}{\lambda d_{b}} \int_{0}^{\lambda} \int_{d_{b}}^{1} C \mathrm{~d} x \mathrm{~d} y
$$

The deformation begins with a modest perturbation of the interface as evident from small increases of $V_{\mathrm{rms}}$ and $e$ in the early stages, followed by an enhanced growth rate indicating the rise of the primary diapir. This behavior is consistent with the linear instability analysis (Ramberg, 1981), which predicts exponential growth rate for an infinitesimal perturbation. In the later stages when the distortion of the interface is severe, the linearin stability proximity breaks down and 


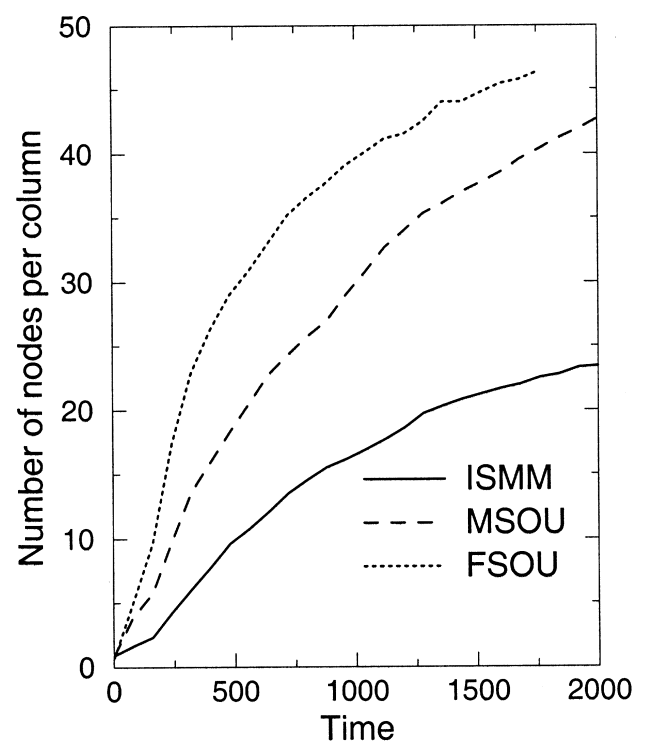

Fig. 6. Average nodal resolving power of the methods for the RT instability of a light layer.

perturbations grow at an increased rate. The rise in $V_{\mathrm{rms}}$ and $e$ values at around $t=600$ is due to the secondary diapir that ascends along the right boundary.

Table 1 gives the initial growth rate of instability, $\gamma$, the maximum value of $V_{\text {rms }}$, the time this maximum value occurs, and the maximum value of $e$ calculated by the three methods used here, as well as those of van Keken et al. (1997). The initial growth rates and maximum $V_{\text {rms }}$ values of our methods are in the range of those predicted by van Keken et al., and the $\gamma$ values closely approximate the analytical value of 0.01094019 for an infinitesimal perturbation (Ramberg, 1981). There are some differences in the time of peak $V_{\text {rms }}$ values and final entrainment at $t=2000$ between our models and those of van Keken et al. The maximum $V_{\text {rms }}$ in our models occurs at about $t=225$ and final entrainment reaches to a value of 0.84 , whereas in their models, the time of the maximum $V_{\text {rms }}$ has an average of $t=207$ and final entrainment is about 0.8 .

\section{Entrainment of the $D^{\prime \prime}$ layer by mantle convection}

In this section, we present results from a set of models designed to study the evolution of the $\mathrm{D}^{\prime \prime}$ layer, a thin dense layer embedded in the lower thermal boundary layer of the convecting mantle. Seismic studies have revealed a great deal about the structure and composition of the $\mathrm{D}^{\prime \prime}$ layer (Lay et al., 1998). The top of the layer is delineated by a seismic discontinuity, and inside the layer, body wave velocity gradients decrease with depth. Regional studies have shown that the thickness of the layer varies from place to place and there is ample evidence for strong lateral heterogeneities within the layer (Kendall and Shearer, 1994). Several mechanisms have been proposed for the origin of compositional heterogeneity of the layer. They include the accumulation of subducted oceanic slabs at the base of the mantle (Olson and Kincaid, 1991) and segregation of the eclogitic component of the slabs (Christensen and Hofmann, 1994), chemical 

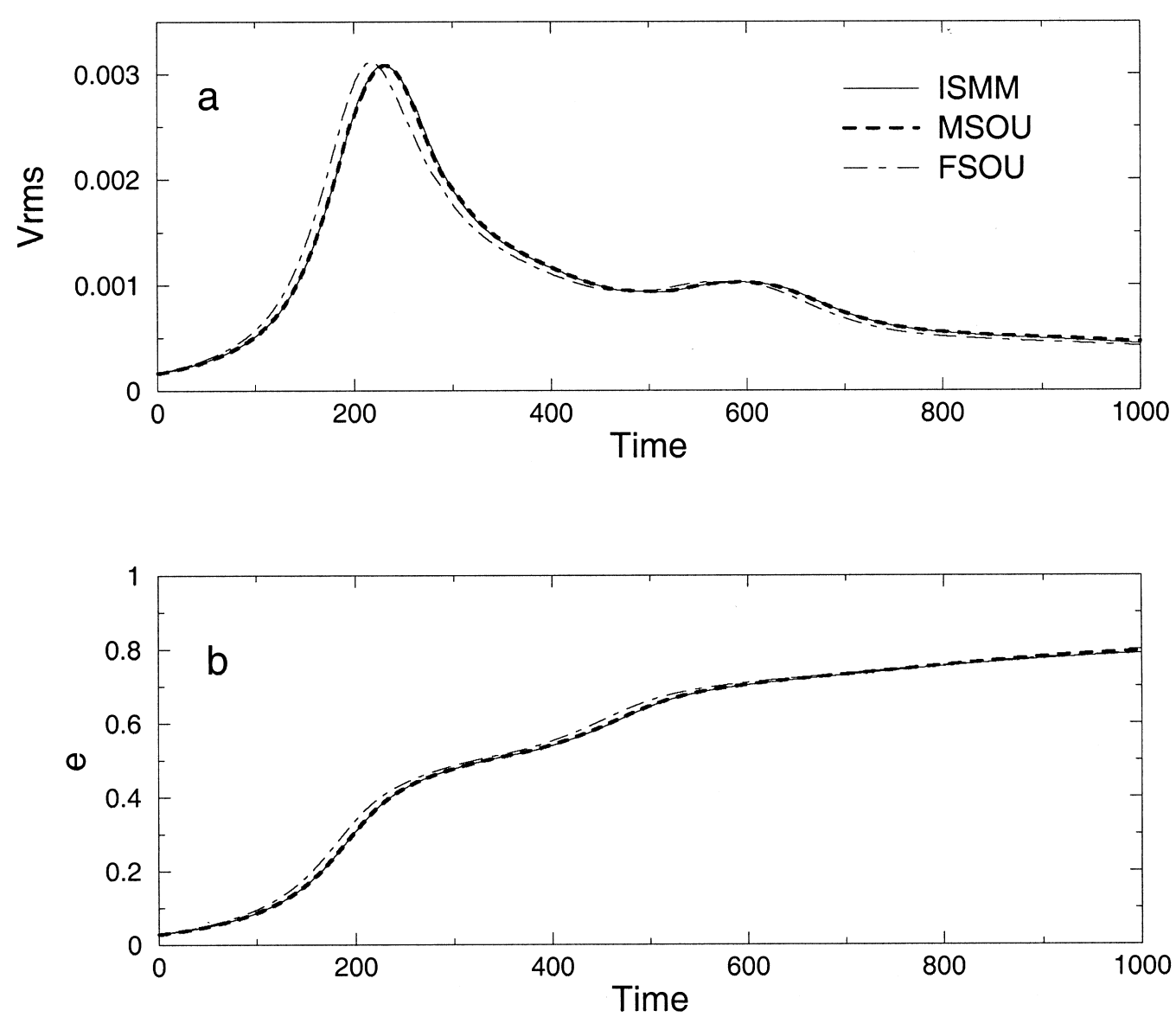

Fig. 7. Comparison of the time variations of global parameters for the RT instability of a light layer. (a) Evolution of $V_{\mathrm{rms}}$, and (b) evolution of the entrainment of the layer. The computations were carried out up to $t=2000$. All models achieved a final $V_{\text {rms }}$ value of 0.00018 and $e$ value of 0.84 .

activity at the CMB between the infiltrated iron from the core and mantle material (Knittle and Jeanloz, 1991), and partial melting and fractionation (Garnero and Helmberger, 1996). Many numerical studies have investigated the mixing of a dense boundary layer with the bulk of the convecting mantle (Hansen and Yuen, 1989; Kellogg, 1997; Montague et al., 1998; Montague and Kellogg, 2000). Most of these studies have concluded that the stability of the dense layer for the most part depends on the density contrast between the layer and the rest of the mantle. For density contrasts of less than $3 \%$, the dense layer is unstable and is completely swept into the mantle. For moderate density contrasts of around 3-6\% the layer can persist for long periods of time, and depending on other factors such as layer thickness and Rayleigh number, lateral variations in the structure of the layer and even small scale convection can develop. For higher density contrasts, the dense layer tends to stabilize at the base of the mantle with little variations in thickness across its length (see Montague and Kellogg, 2000 for a review).

We examine the effects of the layer properties and the Rayleigh number on the degree to which the dense layer can mix with the bulk of the mantle, and also measure the accuracy of our method 
Table 1

Comparison of global parameters of the isothermal RT instability of a thin light layer. The last four rows are taken from the best results of van Keken et al. (1997)

\begin{tabular}{llllll}
\hline Method & Grid size & $\gamma$ & $t_{\max }$ & $\max V_{\text {rms }}$ & $e$ \\
\hline ISMM & $160 \times 160$ & 0.00991 & 230.1 & 0.003093 & 0.84 \\
ISMM & $120 \times 120$ & 0.00998 & 226.1 & 0.003133 & 0.84 \\
MSOU & $160 \times 160$ & 0.00993 & 231.4 & 0.003085 & 0.85 \\
MSOU & $120 \times 120$ & 0.01020 & 227.6 & 0.003134 & 0.85 \\
FSOU & $160 \times 160$ & 0.01111 & 217.3 & 0.003118 & 0.84 \\
FSOU & $120 \times 120$ & 0.01159 & 213.1 & 0.003151 & 0.84 \\
SK & $120 \times 120$ & 0.01127 & 206.4 & 0.002892 & 0.8 \\
HS & $81 \times 81$ & 0.01117 & 208.9 & 0.003092 & 0.8 \\
PvK & $80 \times 80$ & 0.01225 & 207.1 & 0.003091 & 0.8 \\
CND & $48 \times 48$ & 0.01106 & 208.5 & 0.003094 & 0.8 \\
\hline
\end{tabular}

against other available methods. van Keken et al. (1997) studied this problem with a variety of field and tracer methods and used several flow field quantities to compare the results of the methods. We adopt their model properties to make direct comparisons possible. The mantle is assumed to be an isoviscous box of aspect ratio 2 , having a thin dense layer at the bottom. The thickness of the dense layer, $d_{\mathrm{b}}$, is varied from 75 to $300 \mathrm{~km}$. We investigate two thermal Rayleigh number values of $3 \times 10^{5}$ and $3 \times 10^{6}$ and the compositional Rayleigh number is varied from one half to twice of the corresponding thermal Rayleigh number. The density contrast $\Delta \rho$ between the dense layer and the mantle material can be calculated from the ratio of the chemical to thermal Rayleigh numbers. A temperature difference of $2700 \mathrm{~K}$ across the mantle, a thermal expansivity value of $\alpha=1.5 \times 10^{-5} / \mathrm{K}$, and a mantle density of $\rho=4700 \mathrm{~kg} / \mathrm{m}^{3}$, result in a densitycontrast range of $2-8 \%$ in the models. This range can be ranked as small to moderate. Free-slip boundary conditions are used on all boundaries. The temperature is fixed to 0 and 1 at the top and bottom boundaries, respectively, and on the vertical boundaries a zero heat flux condition is assumed. The initial temperature field is based on the boundary layer solution for the four sides of the box.

Fig. 8 shows the time evolution of the temperature and the compositional fields calculated by our method for various models with different density contrasts and dense layer thicknesses and $R a=3 \times 10^{5}$. The first row shows the results for a model with $\Delta \rho=6 \%$ and $d_{\mathrm{b}}=75 \mathrm{~km}$, corresponding to that of van Keken et al. (1997). The initial temperature field establishes a one-cell clockwise flow in the model. This flow field pushes the entire dense layer to the lower left corner of the box, resulting in a pile of dense material at the base of the upwelling plume on the left. A small portion of the accumulated material is entrained upward by the upwelling plume along the left boundary. The formation of the dense material pile results in a strong horizontal density gradient that in turn induces a buoyancy force along the bottom of the model. This buoyancy force creates a counter flow that acts to transfer the dense material and the upwelling plume towards the center of the box, leading to the formation of a two-cell convection in the model. After the establishment of a thermal plume in the middle of the box, the dense material is steadily entrained by the rising flow and is transferred into the cold boundary layers near the top and the sides. Increasing the density contrast to $\Delta \rho=8 \%$ (row f) does not result in any significant change in the behavior of the dense layer, except that the breakup of convection into two cells occurs 
sooner. The denser layer shows even less tendency to mix with the mantle and a lesser amount of material is entrained by the upwelling plume at the center. Decreasing the value of $\Delta \rho$ (rows $\mathrm{d}$ and e), however, results in a significant interaction of the dense layer with the overlying mantle. The amount of entrainment becomes quite high and the dense layer is swept upward by the upwelling plumes and is completely assimilated in the mantle. Effective heat transfer across a convecting layer requires an aspect ratio 1 flow pattern. The flow field inside an aspect ratio 2 box often equilibrates towards a two-cell flow. The above models show that the position of the upwelling plumes always correlates with the position of the thicker regions of the high density material at the bottom, which further accelerates the development of two-cell convection pattern. From the temperature fields at $t=0.05$, it is evident that the flow field varies from completely onecell pattern at $\Delta \rho=2 \%$ to fully two-cell pattern at $\Delta \rho=8 \%$.

The effect of the initial thickness of the dense layer is illustrated in rows a to c of Fig. 8. As the thickness of the layer increases, it breaks up into two piles and this increases the number of upwellings and downwellings and decreases the aspect ratio of the convection cells. The models show that the downwellings are associated with regions of thinner dense layer and the positions of the upwellings correlate with the thicker parts of the dense layer. For the model with $d_{\mathrm{b}}=300$ $\mathrm{km}$, the entire base of the mantle is covered by a stable blanket of dense layer that does not take part in the convection. This gives rise to a thicker thermal boundary layer at the bottom, and a cooler interior, which in turn diminishes the vigor of convection and slows down the rate of dense layer entrainment by the upwelling plumes.

Fig. 9 shows the time evolution of the Nusselt number, $N u$, calculated along the bottom boundary for various models. All models show a strongly time-varying Nusselt number, that suggests large fluctuations with time of the heat flux through the bottom boundary. Models with larger density contrasts have smaller Nusselt numbers. For a fixed dense layer thickness (Fig. 9a and $\mathrm{d}-\mathrm{f}$ ), the pattern of $\mathrm{Nu}$ variations in the initial one-cell flow stage is similar in models with differing $\Delta \rho$ values. This stage roughly corresponds to $t<0.01$ and is associated with the initial overturn of the convective system. After this stage, the Nusselt number decreases with time, duration of which depends on the density contrast of the model. In the case of the model with $\Delta \rho=2 \%$, no reduction in the bottom heat flux is observed in a statistical sense as the entire dense layer is drawn into the mantle. In models with larger $\Delta \rho$ values, the dense layer covers a larger part of the bottom boundary (compare plots at $t=0.03$ in Fig. 8), and decreases the heat flux across the boundary. Towards the end of simulation, the Nusselt number in all models increases. This stage is associated with the decrease in the surface area of the dense layer when all of the dense layer is moved into the base of the upwellings. The blanketing effect of the dense layer on the heat flux through the bottom boundary is better demonstrated in the models with differing layer thicknesses. Fig. 9a-c show that as the initial thickness of the dense layer increases, the variations with time of the Nusselt number become less pronounced and the heat flux approaches a lower and more steady value over time $\left(N u=8\right.$ for a model with $d_{\mathrm{b}}=150 \mathrm{~km}$, and $N u=4$ for a model with $d_{\mathrm{b}}=300 \mathrm{~km}$ ). As a result of decrease in the heat flux across the lower boundary, a thicker thermal boundary layer develops at the bottom, while average temperature in the interior of the mantle drops.

Increasing the Rayleigh number has some important effects on the dynamics of entrainment. In general, the vigor of convection increases at larger Rayleigh numbers, as the upwellings and downwellings increase in number and become narrower at the same time. Fig. 10 shows results of 

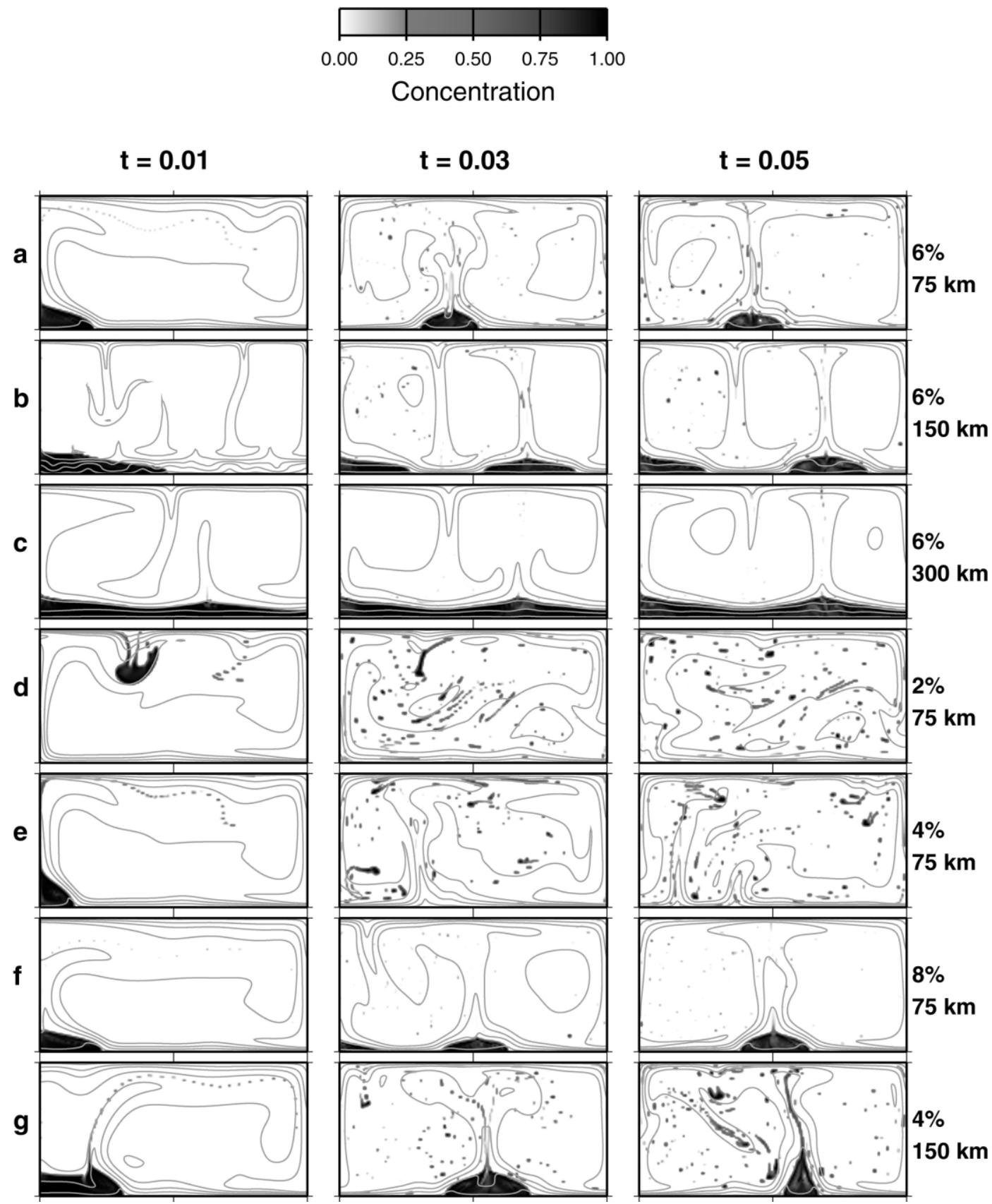

Fig. 8. Time evolution of the composition and temperature fields for various entrainment models with $R a=3 \times 10^{5}$ obtained by our method. Composition field is shown by grey shades. Temperature field is denoted by contour lines with intervals of 0.17. (a) $\Delta \rho=6 \%$ and $d_{\mathrm{b}}=75 \mathrm{~km}$; (b) $\Delta \rho=6 \%$ and $d_{\mathrm{b}}=150 \mathrm{~km}$; (c) $\Delta \rho=6 \%$ and $d_{\mathrm{b}}=300 \mathrm{~km}$; (d) $\Delta \rho=2 \%$ and $d_{\mathrm{b}}=75 \mathrm{~km}$; (e) $\Delta \rho=4 \%$ and $d_{\mathrm{b}}=75 \mathrm{~km}$; (f) $\Delta \rho=8 \%$ and $d_{\mathrm{b}}=75 \mathrm{~km}$; and (g) $\Delta \rho=4 \%$ and $d_{\mathrm{b}}=150 \mathrm{~km}$. 


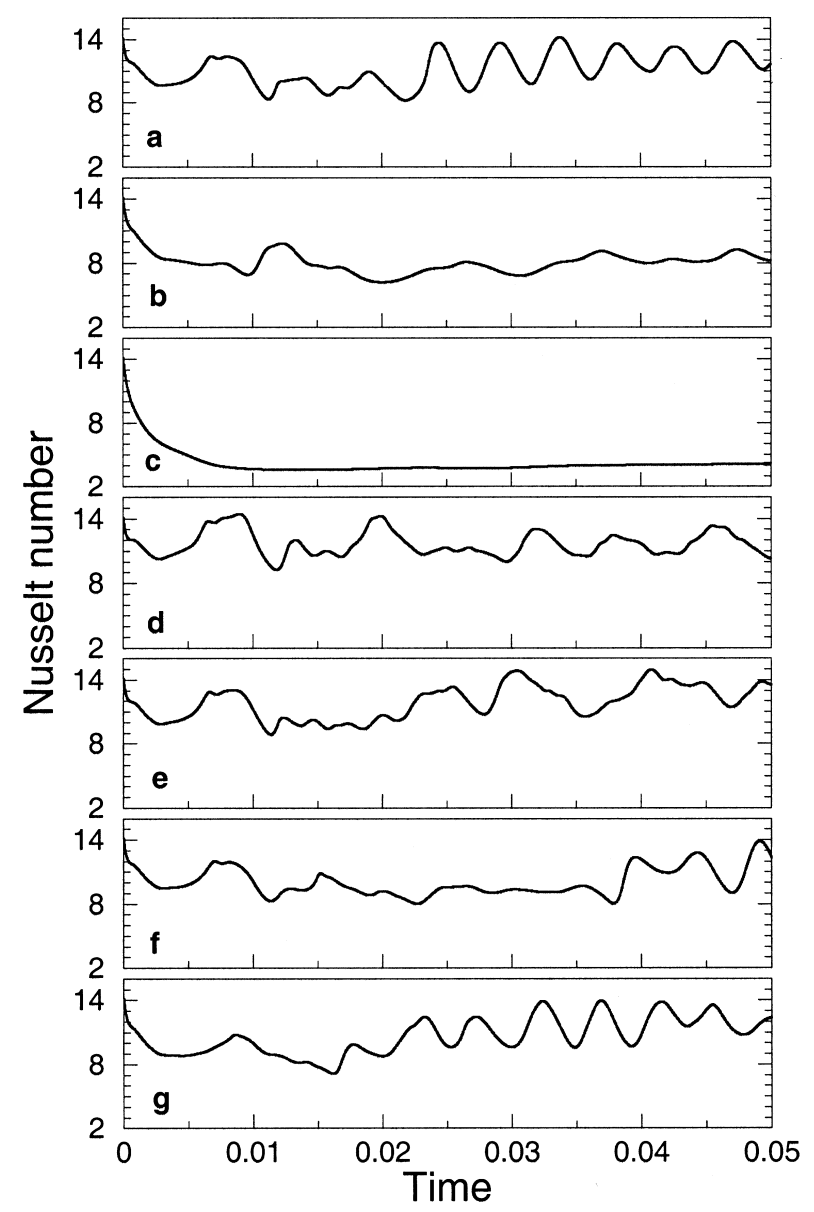

Fig. 9. Time variations of the average Nusselt number in various entrainment models obtained by our method. (a) $\Delta \rho=6 \%$ and $d_{\mathrm{b}}=75 \mathrm{~km} ;$ (b) $\Delta \rho=6 \%$ and $d_{\mathrm{b}}=150 \mathrm{~km} ;$ (c) $\Delta \rho=6 \%$ and $d_{\mathrm{b}}=300 \mathrm{~km} ;\left(\right.$ d) $\Delta \rho=2 \%$ and $d_{\mathrm{b}}=75 \mathrm{~km} ;($ (e) $\Delta \rho=4 \%$ and $d_{\mathrm{b}}=75 \mathrm{~km}$; (f) $\Delta \rho=8 \%$ and $d_{\mathrm{b}}=75 \mathrm{~km} ;(\mathrm{g}) \Delta \rho=4 \%$ and $d_{\mathrm{b}}=150 \mathrm{~km}$.

two models with $R a=3 \times 10^{6}$ and $\Delta \rho=6 \%$, but different initial layer thicknesses. In comparison with the corresponding lower $R a$ models in Fig. 8, there is a greater number of smaller thermal structures inside the mantle, while it becomes increasingly difficult to push the dense layers into piles at the base of mantle plumes. The dense layer evolves into a continuous and stable layer that greatly reduces the heat flux across the bottom boundary. As a result, the interior becomes colder, while the dense layer is heated up. This indicates that at higher $R a$ values, the dense layer stabilizes at even lower density contrasts and at smaller layer thicknesses. This is mainly because of the fact that at higher $R a$ values, the relative magnitude of the viscous to the buoyancy forces is reduced. We achieved a higher $R a$ convection by decreasing the viscosity while keeping the thermal and compositional buoyancy forces unchanged. Consequently, the shear stress exerted by the mantle flow on the top of the dense layer is not great enough to cause significant entrainment of the dense layer at larger density contrasts. An additional feature that is observed in models with higher $R a$, is the small-scale structures inside the dense layer. Fig. 11 shows the horizontally- 

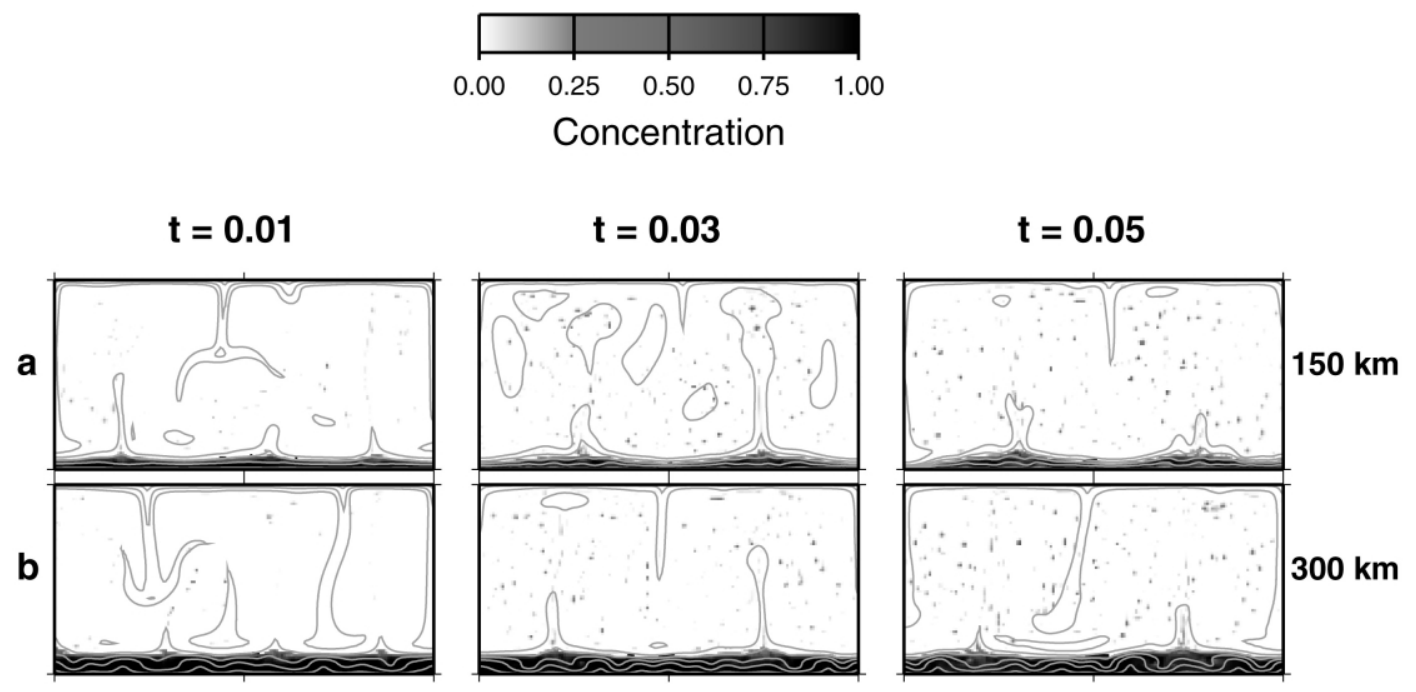

Fig. 10. Time evolution of the composition and temperature fields for two models with $R a=3 \times 10^{6}, \delta \rho=6 \%$, and (a) $d_{\mathrm{b}}=150 \mathrm{~km}$; and (b) $d_{\mathrm{b}}=300 \mathrm{~km}$. Figure specifications are the same as in Fig. 8 .

averaged temperature across the mantle depth for some of the models. The profile with $R a=3 \times 10^{6}$ and $d_{\mathrm{b}}=300 \mathrm{~km}$, shows a double thermal boundary layer at the bottom of the model. This feature is suggestive of a local convection inside the thick dense layer. The existence of an internally convecting stable dense layer is also confirmed by the numerical results of Montague and Kellogg (2000), and the analogue experiments of Olson and Kincaid (1991). This convection pattern is restricted to a narrow range of parameters, and requires density contrasts of $6 \%$ and larger, and layer thickness of at least $300 \mathrm{~km}$.

Fig. 12 shows the results of calculations by the FSOU and MSOU methods for a model with $\Delta \rho=6 \%$ and $d_{\mathrm{b}}=75 \mathrm{~km}$ and of MSOU for a model with $\Delta \rho=8 \%$ and $d_{\mathrm{b}}=75 \mathrm{~km}$. Comparison of results with those of our method in Fig. 8 shows that the numerical methods result in different dynamics, particularly after the breakup of the one-cell flow. In the case of FSOU, the diffusion process is so dominant that the dense material loses its coherence and is smeared out over a large area in the interior of convective cells. The MSOU fields show that most of the material remains inside the thermal upwellings and downwellings and is recycled into the flow along the top and side boundaries. The amount of diffusion is still noticeable, although it is less than that of FSOU. In contrast, our results show that there is little lateral diffusion and only a small portion of the dense material enters the interior of the convective cells. This slow rate of entrainment due to lesser diffusion error is important for the maintenance of the lateral chemical heterogeneity at the bottom of the mantle over a longer period of time.

The entrainment values of our method for a case with $\Delta \rho=6 \%$ and $d_{\mathrm{b}}=75 \mathrm{~km}$ lie in the range of those obtained by van Keken et al. (1997). At $t=0.03$ their marker chain method has an entrainment value of 0.1 and their field and tracer methods give a value of 0.2 , whereas our entrainment values vary from 0.12 to 0.2 over time. Both FSOU and MSOU give very high entrainment values, exceeding 0.5 in the later stages of deformation $(t>0.03)$, owing to their excessive diffusion error. 


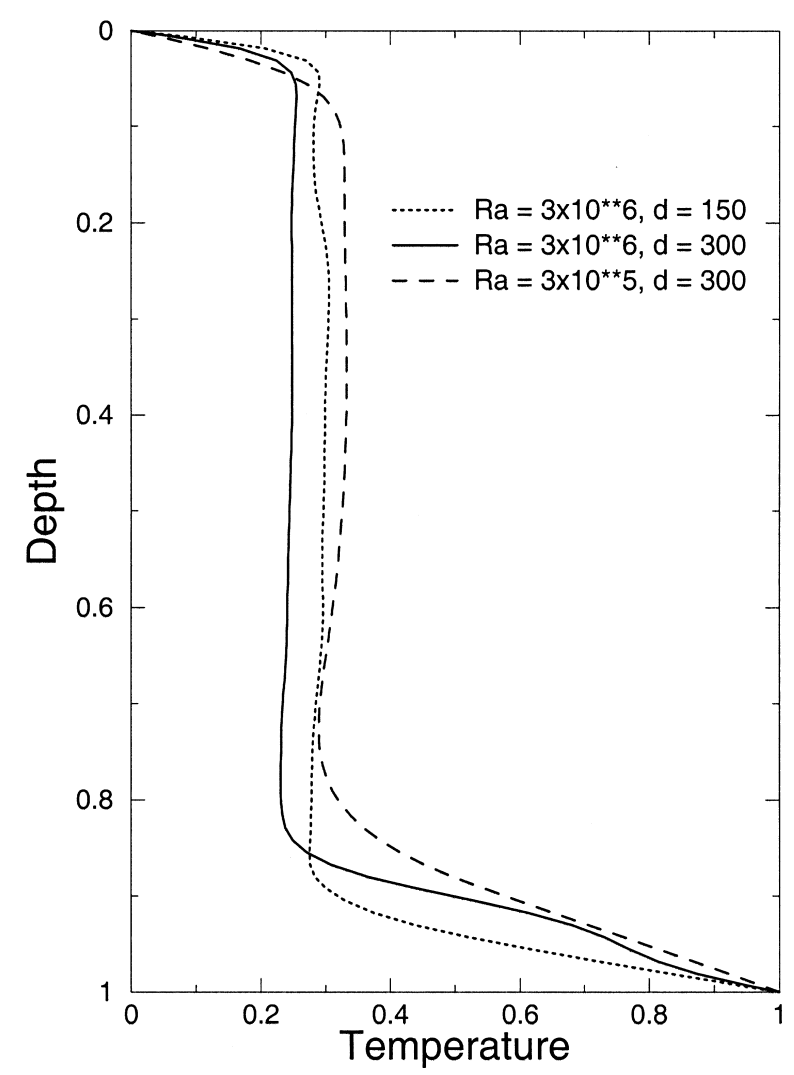

Fig. 11. Profiles of horizontally-averaged temperature of the models at $t=0.05$.

\section{Discussion and conclusions}

As shown in the models of the $\mathrm{D}^{\prime \prime}$ layer entrainment, the density of the $\mathrm{D}^{\prime \prime}$ layer and its thickness have decisive influence on the stability of the layer. Three different patterns of behavior can be discerned in the models. In models with density contrast of less than $4 \%$, the entrainment of the $\mathrm{D}^{\prime \prime}$ is the most severe and it results in complete assimilation of the $\mathrm{D}^{\prime \prime}$ layer in the mantle. In such cases very large thicknesses are required to maintain the stability of the layer and even in that case, the amount of entrainment is high enough to considerably erode the layer over long periods of time. For moderate density contrasts of $6-8 \%$, the $\mathrm{D}^{\prime \prime}$ is stable over long time scales, but shows strong variations in its thickness and has strong tendency to accumulate under mantle upwellings. For this density contrast range, larger layer thicknesses of the order of 200-300 km are required to maintain a continuous layer of dense material at the base of the mantle. As the layer thickness increases, the topographic variations at the top of the layer become less pronounced and more spread out. A consequence of a stable $\mathrm{D}^{\prime \prime}$ layer is the thickening of the thermal boundary layer at the base of the mantle and the drop in the temperature of the mantle plumes. In some of the models, a small-scale convection develops inside the dense layer. This convective pattern can occur if the dense layer is stable enough to decouple from the overlying convecting mantle. Such decoupling requires large density contrasts and thick dense layer, and is more easily 


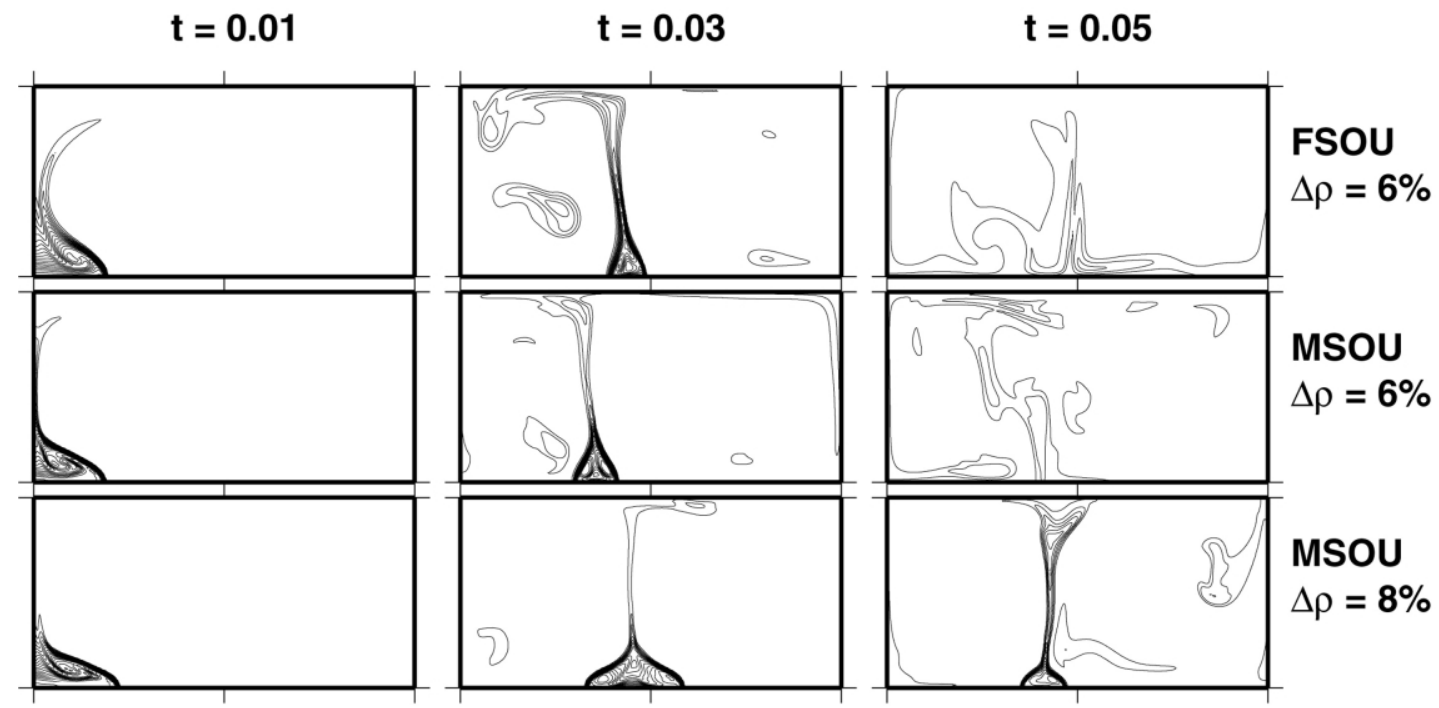

Fig. 12. Time evolution of the composition field of a model with $\Delta \rho=6 \%$ and $d_{\mathrm{b}}=75 \mathrm{~km}$ calculated by FSOU and MSOU, and of a model with $\Delta \rho=8 \%$ and $d_{\mathrm{b}}=75 \mathrm{~km}$ calculated by MSOU.

achieved at higher Rayleigh numbers. These results are well in agreement with those of other investigators. For example, Hansen and Yuen (1989) and Kellogg and King (1993) proposed a density contrast of $2-6 \%$, and Tackley (1998) determined a range of 3-6\% that is needed to maintain the stability of the $\mathrm{D}^{\prime \prime}$ layer. Furthermore, the numerical results of Montague and Kellogg (2000) and analogue experiments of Olson and Kincaid (1991) have demonstrated the development of a second thermal boundary layer near the base of the mantle as a consequence of small-scale convection within the dense layer. There is little indication that the parts of the dense material that are entrained into the mantle, settle out of the convection at later times. The entrained material gradually diffuses away and homogenizes with the bulk of the mantle. The results of our models fit some of the observations as well. For instance seismic evidence suggest the presence of melt at the base of the mantle (Williams and Garnero, 1996). Our models show enhancement of temperatures at the base of the mantle as a result of the presence of the dense layer. Although, we have not incorporated the melting process in our models, higher temperatures within the dense layer may lead to partial melting. Other seismic evidence (Kendall and Shearer, 1995; Kellogg, 1997), suggest that the mantle downwellings are correlated with thinner regions of the $\mathrm{D}^{\prime \prime}$ layer, which is clearly observed in the models.

With regards to the accuracy of our numerical method, the results of the Rayleigh-Taylor instability test problems show that our method outperforms the field methods in maintaining the diffusion error across material discontinuities at a minimal level, and in retaining the step shape of the material interfaces. The results of our method for the second problem are also consistent with the tracer and marker chain methods. The main differences are in the timing of the onset of major instabilities in the flow. It is possible that these differences are partly due to the differences in the accuracy of the various numerical schemes employed in the spatial and temporal discretization of other equations, rather than in the methods used to solve the advection equation for composition. In the case of the $\mathrm{D}^{\prime \prime}$ layer entrainment, much larger differences are seen between our method and the field methods and also tracer and marker chain methods. The results 
suggest that excessive numerical diffusion can lead to wrong estimates of the time that the $\mathrm{D}^{\prime \prime}$ layer can persist at the bottom of the mantle, which in turn influences the outcome of the convection. As confirmed by van Keken et al. (1997), different methods result in different dynamics. We used several spatial resolutions and time integration criteria in order to obtain similar solutions among the methods. We conclude that very high resolutions are required to study details of the evolution of the entrainment process. We used time-step sizes of the order of 0.1 of the Courant time step for the advection of composition field in order to obtain reliable results. van Keken et al. (1997) conclude that the marker chain method is the most accurate among the various schemes they used. However, the cost of tracing and regridding the rapidly growing marker chain during the long-term mixing in thermo-chemical convection renders the method computationally expensive and ultimately impractical. The tracer methods seem to be the most suitable for the study of long-term multicomponent flow problems, although for accurate results they too require a large number of tracers which inevitably increases the computation time. Our method has a relatively low computational cost that is comparable to the field methods which are often more efficient than the tracer and marker chain methods in problems involving extensive mixing of chemical species. For the first instability problem, the ratios of the computation times of FSOU and MSOU to that of our method are 1.62 and 0.98 , respectively. For the second instability problem these ratios are 0.95 and 0.91 , respectively, and for the entrainment problem, 0.85 and 0.88 , respectively. The low computation cost of our method makes it a viable alternative to tracer and marker chain methods for the study of complex geodynamic problems. Furthermore, it is conveniently extendible to three dimensions, whereas the marker chain becomes a marker sheet with serious geometrical difficulties, and the computational cost of the tracer methods prohibitively increases.

\section{Acknowledgements}

This research was supported by a grant from the Natural Sciences and Engineering Research Council (NSERC) of Canada to J.A.H. A.G. and F.S. were partially supported by a scholarship from the ministry of culture and higher education of Iran. We would like to thank M. Nassiri for providing us with his SMM Fortran code. We also thank two anonymous reviewers for their comments and suggestions.

\section{References}

Blankenbach, B. et al., 1989. A benchmark comparison for mantle convection codes. Geophys. J. Int. 98, $23-38$.

Braginski, S.I., 1963. Structure of the F-layer and reasons for convection in the Earth's core. Dokl. Akad. Nauk SSSR $149,8-10$.

Christensen, U.R., 1997. Influence of chemical buoyancy on the dynamics of slab in the transition zone. J. Geophys. Res. 102, 22435-22443.

Christensen, U.R., Hofmann, A.W., 1994. Segregation of subducted oceanic crust in the convecting mantle. J. Geophys. Res. 99, 19867-19884.

Egan, B.A., Mahoney, J.R., 1972. Numerical modeling of advection and diffusion of urban area source pollutant. J. Applied Meteorology 11, 312-322.

Ferrachat, S., Ricard, Y., 1998. Regular vs. chaotic mantle mixing. Earth. Planet. Sci. Lett. 155, 75-86. 
Garnero, E.J., Helmberger, D.V., 1996. Seismic detection of a thin laterally varying boundary layer at the base of the mantle beneath the Central Pacific. Geophys. Res. Lett. 23, 977-980.

Ghods, A., Sobouti, F., Arkani-Hamed, J., 2000. An improved second moment method for solution of pure advection problems. Inter. J. Numer. Methods Fluids 32, 959-977.

Hansen, U., Yuen, D.A., 1989. Dynamical influences from thermal-chemical instabilities at the core-mantle boundary. Geophys. Res. Lett. 16 (7), 629-632.

Hess, P.C., Parmentier, E.M., 1995. A method for the thermal and chemical evolution of the Moon's interior: implications for the onset of mare volcanism. Earth Planet. Sci. Lett. 134, 501-514.

Kellogg, L.H., 1997. Growing the Earth's D" layer: effect of density variations at the core-mantle boundary. Geophys. Res. Lett. 24, 2749-2752.

Kellogg, L.H., King, S.D., 1993. Effect of mantle plumes on the growth of $\mathrm{D}^{\prime \prime}$ by reaction between the core and mantle. Geophys. Res. Lett. 20 (5), 379-382.

Kellogg, L.H., King, S.D., 1997. The effect of temperature dependent viscosity on the structure of new plumes in the mantle: results of a finite element model in a spherical, axisymmetric shell. Earth Planet. Sci. Lett. 148, 13-26.

Kellogg, L.H., Turcotte, D.L., 1990. Mixing and the distribution of heterogeneities in the chaotically convecting mantle. J. Geophys. Res. 95, 421-432.

Kendall, J.M., Shearer, P.M., 1994. Lateral variations in $\mathrm{D}^{\prime \prime}$ thickness from long-period shear-wave data. J. Geophys. Res. 99, 11575-11590.

Kendall, J.M., Shearer, P.M., 1995. On the structure of the lowermost mantle beneath the southwest Pacific, southeast Asia and Australasia. Phys. Earth. and Planet. Int. 92, 85-98.

Knittle, E., Jeanloz, R., 1991. Earth's core-mantle boundary: results of experiments at high pressure and temperatures. Science 251, 1438-1443.

Lay, T., Williams, Q., Garnero, E.J., 1998. The core-mantle boundary and deep Earth dynamics. Nature 392, $461-468$.

Lenardic, A., Kaula, W.M., 1993. A numerical treatment of geodynamic viscous flow problems involving the advection of material interfaces. J. Geophys. Res. 98, 8243-8260.

Lenardic, A., Kaula, W.M., 1996. Near surface thermal/chemical boundary layer convection at infinite Prandtl number: two-dimensional numerical experiments. Geophys. J. Int. 126, 689-711.

Montague, N.L., Kellogg, L.H., Manga, M., 1998. High Rayleigh number thermo-chemical models of a dense boundary layer in $\mathrm{D}^{\prime \prime}$. Geophys. Res. Lett. 25, 2345-2348.

Montague, N.L., Kellogg, L.H., 2000. Numerical models of a dense layer at the base of the mantle and implications for the geodynamics of $\mathrm{D}^{\prime \prime}$. J. Geophys. Res. 105, 11101-11114.

Olson, P., Kincaid, C., 1991. Experiments on the interaction of thermal convection and compositional layering at the base of the mantle. J. Geophys. Res. 96, 4347-4354.

Patankar, S.V., 1980. Numerical Heat Transfer and Fluid Flow. Hemisphere Publishing (197 pp.).

Ramberg, H., 1981. Deformation of the Earth's Crust in Theory, Experiments and Geologic Applications, 2nd Edition. Academic (425 pp.).

Sweby, P.K., 1984. High resolution schemes using flux limiters for hyperbolic conservation laws. SIAM J. Numer. Anal. 21, 995-1001.

Tackley, P.J., 1998. Three-dimensional simulations of mantle convection with a thermo-chemical basal boundary layer: $\mathrm{D}^{\prime}$. In: Gurnis, M. et al. (Eds.), The Core-Mantle Boundary Region, Vol. 28, Geodynamic Series. AGU, Washington, DC, pp. 231-254.

Tamamidis, P., Assanis, D.N., 1993. Evaluation of various high-order-accuracy schemes with and without flux limiters Inter. J. Num. Methods, Fluids 16, 931-948.

van Keken, P.E., King, S.D., Schmeling, H., Christensen, U.R., Neumeister, D., Dion, M.P., 1997. A comparison of methods for the modeling of thermochemical convection. J. Geophys. Res. 102, 22477-22495.

van Keken, P.E., Spiers, C.J., van den Berg, A.P., Muyzert, E.J., 1993. The effective viscosity of rock salt: implementation of steady state creep laws in numerical models of salt diapirism. Tectonophysics $225,457-476$.

Whitehead, J.A., Luther, D.S., 1975. Dynamics of Laboratory diapirand plume models. J. Geophys. Res. 80, $705-717$.

Williams, Q., Garnero, E.J., 1996. Seismic evidence for partial melt at the base of the Earth's mantle. Science 273, 1528-1530.

Woidt, W.D., 1978. Finite element calculations applied to salt dome analysis. Tectonophysics 50, 369-386. 